Copyright (c) 2012 Universidad Tecnológica Equinoccial

ISSN: $1390-6542$

\title{
Teoría de restricciones para procesos de manufactura
}

\author{
Ing. Gabriela Villagómez ${ }^{1}$, Ing. Jorge Viteri, MBA-M.Sc. ${ }^{1}$, Dr. Alberto Medina, Ph.D. ${ }^{2}$
}

\section{Resumen}

La empresa "Productos Alexander" fabricante de snacks de la ciudad de Quito, tiene un sistema de producción que no permite el abastecimiento de los pedidos de sus clientes reflejando un índice de servicio al cliente de $89,58 \%$ ya que sus procesos están trabajando con capacidades que no se encuentran correctamente programadas, en vista de tal razón el objetivo de esta investigación es proponer un sistema de mejora continua con el fin de aumentar y efectivizar específicamente la producción de snacks de papas fritas utilizando la Teoría de Restricciones (TOC) como metodología científica. Se realizó la medición de capacidad de cada una de las operaciones que componen el proceso de elaboración de snacks y se identificó la restricción en el proceso de fritura, que no estaba trabajando el 100\% del tiempo en la jornada diaria limitando así la capacidad de la fábrica, por lo que se optó por explotar la restricción para eliminarla. La propuesta planteada para explotar la restricción consiste en una mejor programación de la producción, reducción de lote de transferencia de proceso a proceso, programación de la cantidad de trabajo para cada uno de los operarios en la jornada, la preparación del proceso y la fijación de turnos en la hora de almuerzo de los operarios lo que contribuye a agilizar el proceso de fritura y permitir que trabaje a su máxima capacidad el 100\% del tiempo.Dicha propuesta se puede considerar como una solución al problema y así mejorar los niveles de producción de la empresa, tomando en cuenta que en base a los indicadores de TOC el Beneficio Neto de la empresa aumentaría de \$15 333.09 a \$ 17392.14 al cumplir en cantidad y a tiempo todos los pedidos de los clientes.

\section{Palabras c lave}

Sistema, restricción, explotación, mejora continua, Beneficio Neto.

\section{Abstract}

The company "Products Alexander" snack manufacturer in the city of Quito, has a production system that cannot supply customer orders, with a customer service index of $89,58 \%$, also their processes are working with capabilities that are not properly programmed in given this reason the objective of this research is to propose a system of continuous improvement to increase production and make effective the snacks of chips using the Theory of Constraints (TOC) as a scientific methodology is to focus on solutions to critical problems of enterprises so that they approach their goal through a process of continuous improvement (Arias, 2006). The measurement was carried capacity of each of the operations which compose the preparation of snacks and identified restriction in the frying process, which was not working $100 \%$ of the daily time thereby limiting the ability of the factory, so we chose to exploit the constraint to eliminate

\footnotetext{
${ }^{1}$ Universidad Tecnológica Equinoccial, Facultad de Ciencias e Ingeniería, Quito-Ecuador (jviteri@ute.edu.ec)

${ }^{2}$ Universidad de Matanzas Camilo Cienfuegos, Cuba
} 
it. The proposal made to exploit the constraint is a better production scheduling, reducing transfer batch process to process, scheduling the amount of work for each of the operators in the day, the preparation process and setting shifts at lunchtime for workers helping to streamline the process of frying and allow work to full capacity $100 \%$ of the time. This proposal can be considered as a solution to problems and improve production levels of the company, considering that based on indicators of TOC net income the company would increase from $\$ 15$ $333.09 \$ 17392.14$ in meeting quantity time and all customer orders.

Keywords

System, restriction, exploitation, continuous improvement, net benefit

\section{Introducción}

Todas las empresas buscan implementar un proceso de mejoramiento continuo exitoso y perdurable, puesto que si no se ajustan a las tendencias actuales no pueden incrementar la eficiencia y productividad en sus procesos, además no logran generar ventajas competitivas que les garanticen una permanencia sostenible en el mercado ni mucho menos su crecimiento y desarrollo.

Según Viteri et al. (2011) para que un país sea competitivo es necesario aplicar las ideas innovadoras en hechos concretos que se traduzcan en beneficios tangibles, económicamente viables y sostenibles en el tiempo; es decir dar un salto de las ideas a la realidad, de pensar a hacer.

Por ello es necesario la realización de un estudio del proceso de elaboración de snacks basado en la Teoría de Restricciones para su futura implementación, en función de su situación actual y con la finalidad de determinar la restricción del sistema y proponer mejoras que le permitan explotar la misma y trabajar conjuntamente para alcanzar sus metas y objetivos globales.

Según Aguilar y Vargas (2008) en México se reportó que los problemas más comunes a los que se enfrentan las pequeñas empresas se refieren al mal manejo del personal, medio ambiente, mal manejo de los recursos financieros, deficientes métodos de trabajo, problemas en la maquinaria y equipo y subutilización de los recursos materiales. Además dentro de los problemas de deficientes métodos de trabajo se encuentran liderando los siguientes: producción, ventas, control y evaluación de las actividades de los operarios.

En el caso de la empresa "Productos Alexander" el proceso de elaboración de snacks está trabajando con capacidades que no están correctamente programadas lo que le impide crecer y alcanzar mayores niveles de productividad, sin poder abarcar grandes mercados ni satisfacer a los clientes para garantizar su permanencia y desarrollo sostenible.

Cuando la demanda de snacks sobrepasa la producción semanal, no se logra entregar pedidos completos a los clientes y en ciertas ocasiones se lo hace a destiempo, limitada así por falta de planificación de su capacidad de producción y los incrementos de la demanda de sus productos. 
El proyecto de elaborar una propuesta de mejora para el proceso productivo de elaboración de snacks obedece a que existe una restricción en el sistema que le impide cumplir el 100\% de los pedidos de sus clientes y que provoca también un retraso en ciertas entregas.

El proceso tiene un problema potencial de programación de su capacidad, por esta razón es necesario que esté dispuesto a considerar las mejoras propuestas para la explotación de la restricción determinada en base a TOC, pensando que de esta manera puede alcanzar sus objetivos organizacionales, adaptarse a los cambios dados por la globalización y las nuevas exigencias del mercado así como el incremento de su productividad y competitividad en el mismo.

La Teoría de Restricciones (TOC) es todo un proceso de mejoramiento continuo, basado en un pensamiento sistémico, que ayuda a las empresas a incrementar sus utilidades con un enfoque simple y práctico, identificando las restricciones para lograr sus objetivos, y permitiendo efectuar los cambios necesarios para eliminarlos (Goldratt, 1993).

De acuerdo a Aguerre (2009), para desarrollar el proceso de mejora continua propuesto por Goldratt, la Teoría de Restricciones se basa en el siguiente ciclo compuesto por cinco pasos:

1. Identificar la restricción del sistema

2. Decidir cómo explotar la restricción del sistema

3. Subordinar todas las actividades del sistema.

4. Elevar la restricción del sistema.

5. Implementar y volver a analizar el sistema.

Para Goldratt (1993), la Teoría de Restricciones reconoce que la producción de un sistema consiste en múltiples pasos, donde el resultado de cada uno de esos pasos depende del resultado de pasos previos. El resultado o la producción del sistema, estará limitada por el o los pasos menos productivos.

Espinoza y Jiménez (2007) concluyen que una restricción conocida también como cuello de botella, se define como cualquier elemento que está limitando al sistema en el cumplimiento de la meta para la que fue creado, es decir, para el caso de empresas con fines de lucro, generar ganancias sustentables.

Se distinguen dos tipos de recursos productivos:

- Recurso Restricción (RRC): es aquel cuya capacidad es menor o igual a la demanda que hay de él.

- Recurso no Restricción: es aquel cuya capacidad es mayor que la demanda que hay de él. 
De acuerdo a Berrío y Castrillón (2008), cuando un sistema no puede generar más rentabilidad es porque algo se lo está impidiendo, esto es debido a las restricciones que pueden ser una persona, un equipo, una máquina, una pieza, una política de la empresa, la ausencia de alguna herramienta de apoyo, etc.

Las restricciones no son negativas ni positivas, son una realidad puesto que en una gran cadena de recursos interdependientes solo unos pocos de ellos, los cuellos de botella (o restricciones) condicionan la salida de toda la producción, es por ello que hay que utilizarlos para manejar el flujo del sistema productivo (Escalona, 2009).

Según Goldratt (1993), lo que determina la capacidad de la planta es la capacidad del recurso con restricción de capacidad, por lo que se debe balancear el flujo de todos los recursos productivos al ritmo del cuello de botella y aprovechar el tiempo al máximos pues una hora perdida en este tipo de recursos es una hora perdida en todo el sistema productivo.

Las empresas que utilizan la Teoría de Restricciones como herramienta para el mejoramiento continuo de sus procesos logran fortalecer su competitividad a nivel de calidad, servicio al cliente y bajo costo; logran también la reducción en el tiempo de entrega, mejora en el cumplimiento de las fechas de entrega, reducción en los inventarios, incremento de las ventas y el incremento de las utilidades netas (Morales, 2006).

A nivel general, la Teoría de Restricciones se desarrolla en procesos de pensamiento así como en aplicaciones a distintas áreas entre las cuales se distinguen la producción, finanzas, administración de proyectos, marketing, ventas, sistemas de distribución, recursos humanos, entre otros (Herrera, 2003).

Es importante contar con la propuesta de mejoramiento que plantea la Teoría de Restricciones que le permita al proceso de elaboración de snacks identificar su restricción y atacarla a través de la explotación de la misma.

En base a Aguerre (2009), para explotar la restricción es preciso sacarle lo que más se pueda al recurso restricción o cuello de botella sin perder tiempo, es decir, debemos obtener el máximo rendimiento del recurso con restricción de capacidad dentro de las 8 horas laborables pues se conoce que cualquier minuto perdido en el rendimiento del recurso con restricción de capacidad repercute en el nivel de producción de todo el sistema.

Existe una técnica que permite disminuir los tiempos de alistamiento rápido conocida como Single Minute Exchange of Die (SMED) (Olavarrieta, 1999).Las siglas SMED al español se traduce como Reducción de Tiempo de Preparación de Máquina y se refiere al hecho de efectuar el cambio de 
herramienta o a su vez la preparación de procesos, en un número de minutos que se exprese con un solo dígito, es decir, nueve minutos o menos (Cruz, 2009).

La reducción del lote de transferencia definido como la cantidad de unidades que pasan a la siguiente operación, es otra manera de explotar la restricción ya que si éste se reduce y es el mismo para cada operación, se necesita menos tiempo de procesamiento total en el sistema y se logra disminuir el inventario en proceso lo que a su vez contribuye a agilizar de alguna manera el proceso y resolver problemas de manera rápida.

El balance de flujo del proceso de elaboración de snacks contribuye a explotar la restricción puesto que se optimiza el tiempo y la capacidad logrando de esta manera romper la restricción aumentando la producción y cumpliendo con todos los clientes.

La continuidad en la búsqueda de la mejora requiere de un sistema de medición y de un método que involucre y fomente la participación del personal. Para definir el sistema de medición se requiere definir el sistema de indicadores de la meta. En TOC, la meta de una empresa es ganar dinero ahora y siempre (Escalona, 2009).

De acuerdo a Goldratt (1993), la medición de la meta se realizará a través de los indicadores como el Trúput (T), Gastos Operativos (GO), Inventarios (I).

\section{Trúput $(\mathrm{T})^{1}$}

De acuerdo a Goldratt (2002), se define como: La velocidad a la cual el sistema genera dinero a través de las ventas. Mide cuánto dinero genera el sistema tomando en cuenta:

- Utilidades a través de ventas

- Ingresos como intereses cobrados

- Regalías por patentes, etc.

El Trúput asociado a un producto se define matemáticamente con la siguiente fórmula:

$$
T=N(P V-C T V)
$$

Siendo:

T: Trúput

$\mathrm{N}$ : Cantidad de unidades cobradas en un período.

PV: Precio de venta del producto

\footnotetext{
${ }^{1}$ Trúput es la versión abreviada de Throughput.
} 
CTV: Costos Totalmente Variables. Son aquellos que aumentan de manera directamente proporcional con el volumen de ventas, tales como materias primas y componentes, servicios de terceros, comisiones por ventas, pago por proyecto, etc.

\section{Gastos Operativos (GO)}

Es todo el dinero que el sistema gasta en convertir el inventario en Trúput. (Goldratt, 2009). Son todos los gastos directamente proporcionales con las ventas. Es decir los gastos en los que la empresa incurre aunque no venda. Por ejemplo: sueldos y jornales (semanales, mensuales, etc.), amortizaciones, arrendamiento, materias primas, cuotas de préstamos, pagos de servicios públicos, etc. (Herrera, 2003).

Los Gastos Operativos tienen la siguiente fórmula:

$$
G O=S U E L D O S+\text { GASTODEFABRICACIÓN [2] }
$$

\section{Inventario (I)}

Es todo el dinero que el sistema invierte en elementos que se propone vender (Krajewski et al. 2008). Inventario es el dinero almacenado o retenido dentro del sistema. Algunos componentes del inventario son: stocks de materias primas, de material en proceso y de productos terminados, edificios propios, maquinaria, dinero en efectivo, patentes, clientes por cobrar, etc.

Así definida, la Inversión consiste en todos aquellos elementos que pueden transformarse en dinero mediante su venta.

TOC sostiene que cualquier valor que se asigne a estos elementos es inexacto, ya que solo cuando alguien los compra se sabe cuánto valen realmente.

Los criterios de evaluación de Inversión que usa TOC están enfocados, al igual que todas las medidas de desempeño definidas en la metodología, a inducir a las personas de la organización a actuar según lo que es bueno para el sistema y a disuadirlas de actuar según lo que es malo para el sistema. Estos inventarios son también conocidos como Inversiones operativas (Iglesias, 2000)

\section{Beneficio Neto}

Su fórmula corresponde a la sumatoria de todo el Trúput de un período, por ejemplo 1 mes y la resta de todos los Gastos Operativos correspondientes a ese periodo. (Iglesias, 2000)

$$
\text { BENEFICIO NETO = TRÚPUT - GASTOSOPERATIVOS }
$$

\section{ROI}

Otro indicador que relaciona la utilidad con la inversión es el Retorno sobre la Inversión (ROI), que se calcula así:

$$
\text { ROI = BENEFICIO NETO } \div \text { INVERSIÓN }(\text { INVENTARIOS })
$$


Sin embargo si hay mucho beneficio y un alto ROI, no es razón para quedarse tranquilo puesto que si no hay disponibilidad de efectivo es difícil que la empresa esté saludable. (Agudelo \& Escobar, 2010)

Para todo esto, se plantea como objetivo el identificar la restricción del proceso productivo de elaboración de snacks y elaborar una propuesta para la mejora continua en virtud de explotarla utilizando TOC.

\section{Materiales y métodos}

Con la finalidad de identificar la restricción del sistema se partió de la aplicación de la técnica de observación del proceso productivo de elaboración de snacks, de los fenómenos y hechos que ocurren en cada operación del mismo.

Con el mismo propósito, durante diez días de producción en la planta y aplicando en lotes aleatorios cada día se midió el tiempo de duración y la capacidad en $\mathrm{kg} / \mathrm{min}$ de las operaciones que componen el proceso de snacks según corresponda, para determinar la capacidad promedio de cada una de estas lo cual permitió encontrar la restricción del sistema.

Para cumplir por completo con el objetivo de la investigación se buscó la alternativa más adecuada como propuesta para mejorar el proceso de elaboración de snacks en la cual se explotó la restricción a través de un balance de flujo, de la reducción del lote de transferencia y trabajo $100 \%$ del tiempo en la restricción.

En base a datos proporcionados por la empresa, se determinó el Trúput actual generado por el proceso de elaboración de snacks y un Trúput proyectado como indicador de TOC en el caso de aplicar la propuesta.

\section{Resultados y discusión}

A continuación en la tabla 1, se presenta los datos obtenidos de la duración y capacidad de cada uno de los procesos para encontrar la restricción del sistema.

Tabla 1. Capacidad de los procesos que componen la elaboración de snacks

\begin{tabular}{|l|c|c|c|}
\hline \multicolumn{1}{|c|}{ Proceso } & Duración (min) & Lote de producción (kg) & Capacidad (kg/min) \\
\hline Pelado manual & 2.84 & 8.75 & $\mathbf{3 . 0 1}$ \\
\hline Pelado automático & - & - & $\mathbf{2 . 8 6}$ \\
\hline Rebanado & - & - & $\mathbf{4 . 6 0}$ \\
\hline Fritura & 7.28 & 8.75 & $\mathbf{1 . 2 0}$ \\
\hline Empaque & - & - & $\mathbf{0 . 5 2}$ \\
\hline Sellado & - & - & $\mathbf{0 . 7 2}$ \\
\hline
\end{tabular}


En la figura № 1 se muestra en resumen la capacidad de cada operación del proceso de elaboración de snacks y se identifica la posible restricción.

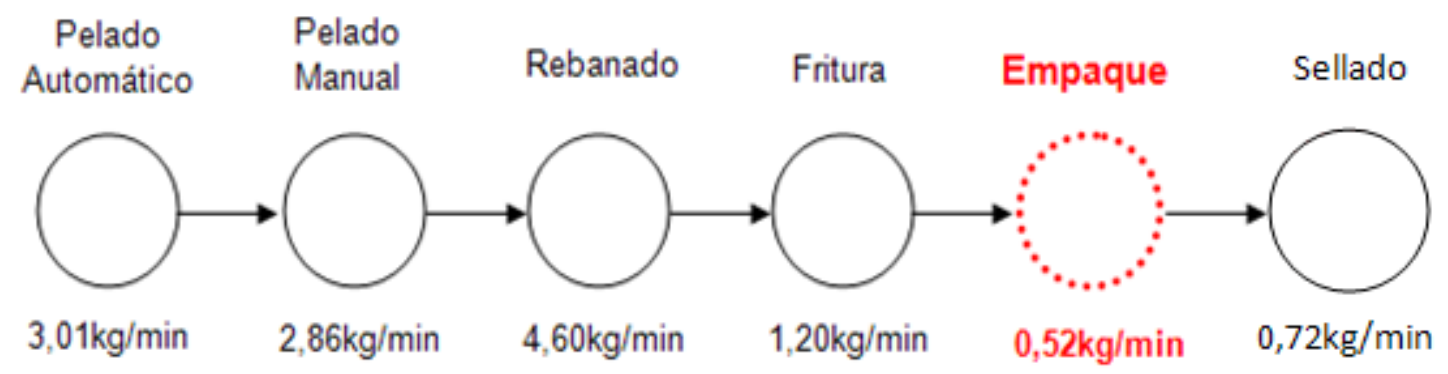

Figura 1. Posible proceso cuello de botella del sistema

En relación a la figura anterior, aparentemente el recurso con restricción de capacidad es el empaque manual, pero es en este punto es necesario tomar en cuenta el rendimiento de la materia prima durante el proceso de elaboración de snacks.

Actualmente se procesan $363.3 \mathrm{~kg}$ diarios de los cuales se obtienen aproximadamente 400 fundas de $200 \mathrm{~g}$ de snakcs de papas fritas, esta cantidad de producto se adquiere después del proceso de fritura y corresponde al $22 \%$ de rendimiento que se ha obtenido de la materia prima.

Se justifica que el proceso de empaque manual no es la restricción del sistema puesto que debido al rendimiento de la materia prima, a esta operación llega una cantidad de producto mucho menor a la que ha sido procesada por las operaciones anteriores de pelado automático, pelado manual, rebanado y fritura, por esta razón tanto el empaque como el sellado tienen capacidad suficiente para llevarse a cabo.

El proceso de fritura se determinó como restricción del sistema ya que restringe la salida de toda la producción de la empresa al no trabajar al 100\% del tiempo de la jornada, además se conoce que la demanda del mercado es mayor a la capacidad que tiene este recurso, y todo esto lo hace responsable de no dar abasto al sistema para poder entregar la totalidad de los pedidos de los clientes.

Según Goldratt (1993), el primer indicador financiero de la Teoría de Restricciones es el Trúput. Para determinarlo lo primero que debemos conocer es el precio de venta del producto, determinando que el precio de venta es de $\$ 1.75$ para una funda de snacks de papas fritas de $200 \mathrm{~g}$.

Los costos totalmente variables son otro dato importante para determinar el Trúput los mismos que conoceremos a continuación.

Costo de 200 g de papas: $\$ 0.60$ 
Costo de una funda: $\quad \$ 0.04$

Total CTV: $\quad \$ 0.64$

Conociendo estos datos se puede determinar el Trúput unitario tomando en cuenta el precio de venta que se ha determinado anteriormente.

$$
\begin{gathered}
T_{1=} 1.75-0.64 \\
T_{1=} 1.11
\end{gathered}
$$

El Trúput del año 2011 para expresar las utilidades de la empresa a través de las ventas de snacks, mediante la siguiente fórmula ya conocida.

$$
\begin{gathered}
T=N(P V-C T V) \\
T=87218(1.75-0.64) \\
T=96811.98
\end{gathered}
$$

El siguiente indicador financiero propuesto por la Teoría de Restricciones corresponde a los Gastos Operativos que se define como la mano de obra a lo que hay que adicionar el costo de fabricación, son los gastos en los que incurre la empresa aunque no venda.

Los Gastos Operativos ascienden a \$ 80907.24.

Para conocer los Gastos Operacionales anuales, se debe tomar en cuenta también las tareas extra se han realizado durante el año que de acuerdo a políticas de la empresa corresponde al procesamiento de $45.45 \mathrm{~kg}$ que se realiza para poder completar los pedidos, cuyo costo se detalla a continuación en la tabla 2.

Tabla 2. Tareas extra realizadas en el año 2011

\begin{tabular}{|l|c|c|c|}
\hline \multicolumn{1}{|c|}{ Mes } & Número de "tareas extra" & Costo unitario (\$) & Costo total (\$) \\
\hline Enero & 19 & 5.55 & 105.45 \\
\hline Febrero & 38 & 5.55 & 210.90 \\
\hline Marzo & 18 & 5.55 & 99.90 \\
\hline Abril & 20 & 5.55 & 111.00 \\
\hline Julio & 8 & 5.55 & 44.40 \\
\hline \multicolumn{3}{|r|}{ Total } & $\mathbf{5 7 1 . 6 5}$ \\
\hline
\end{tabular}

Fuente: Empresa "Productos Alexander"

Tomando en consideración lo anterior los Gastos Operativos (80907.24 + 571.65) corresponden a $\$ 81478.89$. 
Estos indicadores que se han logrado determinar con los datos de la empresa y nos permiten conocer el Beneficio Neto del año 2011.

\author{
BENEFICIO NETO $=$ TRÚPUT - GASTOSOPERATIVOS \\ BENEFICIO NETO $=96811.98-81478.89$
}

BENEFICIO NETO $=\$ 15333.09$

Para explotar la restricción se optó por una técnica que permite disminuir los tiempos de alistamiento rápido conocida como SMED.

La idea de la explotación es que la restricción trabaje Al 100\% del tiempo tomando en cuenta las 8 horas de la jornada diaria, por ello es importante tomar en consideración ciertos elementos necesarios que permitan preparar el proceso de fritura, lo que implica que una vez que las papas cumplan su tiempo de escurrido y pasen a la operación de fritura, ésta se debe llevar a cabo de manera continua, para ello es preciso limpiar y desinfectar la freidora, colocar el aceite y encenderla para precalentarlo en un rango de $160^{\circ} \mathrm{C}-180^{\circ} \mathrm{C}$ de temperatura, lo cual se hace una sola vez al iniciar la jornada de trabajo y toma un tiempo de 15 a 20 minutos.

En este caso SMED propone reducir el tiempo de preparación de este proceso de fritura, precalentando el aceite antes de que la materia prima esté lista para ingresar a dicho proceso, es decir, si todo el proceso inicia a las $08 \mathrm{~h} 25$, es a esa hora también que se debe colocar el aceite en la freidora y precalentarlo a la temperatura óptima con la finalidad que al momento que la materia prima llegue a esta operación se pueda iniciar sin retrasos.

Conociendo que la capacidad del proceso de fritura es de $1.20 \mathrm{~kg} /$ minuto, la producción de esta operación en la jornada de 8 horas debería ser de $576 \mathrm{~kg}$, actualmente se procesan $363.3 \mathrm{~kg}$ por lo tanto, esta operación de fritura está produciendo durante 5 horas de la jornada solamente, existiendo así una pérdida de 3 horas de producción, razón que está ocasionando el incumplimiento del $100 \%$ de los pedidos a los clientes.

Para mejorar la producción en la jornada se propone también la posibilidad de trabajar con lotes de transferencia más pequeños, mejorar el tiempo y la capacidad de producción así como programar el trabajo de los operarios para garantizar el aumento de la producción y responder rápidamente a cambios en la demanda del mercado lo que a su vez permite mejorar el porcentaje de cumplimento a los clientes cuando existan pedidos grandes.

En la figura № 2, se presenta la situación actual del proceso tomando en cuenta el lote de transferencia, su duración en cada operación y la carga de trabajo para cada operario. 


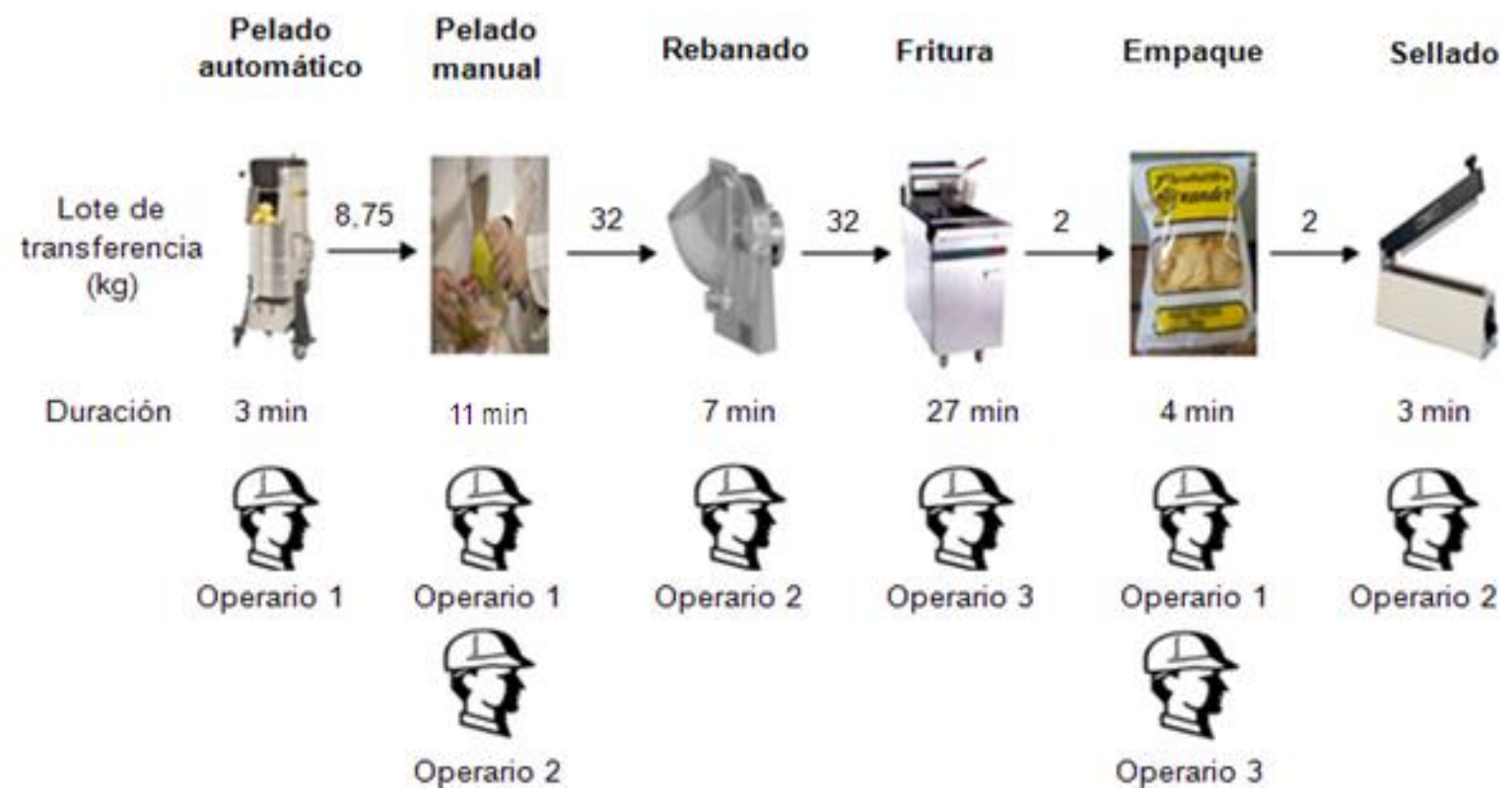

Figura 2.Situación actual del proceso de elaboración de snacks

Para incrementar el Trúput es preciso trabajar todo el tiempo posible en la restricción del sistema, esto se lo hace a través de una mejor programación de la producción donde el proceso de fritura tiene una duración de 8 minutos, es decir, que cada operación se repite nuevamente cada 8 minutos con la finalidad de que este proceso no se quede desabastecido utilizando un lote de transferencia ideal de $9 \mathrm{~kg}$ fijado para todas las operaciones ya que la capacidad máxima de la restricción es de $9 \mathrm{~kg}$ y lo que se busca es que ésta trabaje utilizando toda su capacidad y el mayor tiempo posible durante la jornada lo que a su vez contribuye a agilizar de alguna manera el proceso y resolver problemas de manera rápida, se ha propuesto también la programación de actividades para cada uno de los operarios dentro del proceso lo que permite fijar turnos para el almuerzo de los mismos evitando que el proceso de fritura se detenga.

En la figura № 3, se resume la propuesta para explotar la restricción encontrada en el sistema y permitir la mejora continua del proceso.

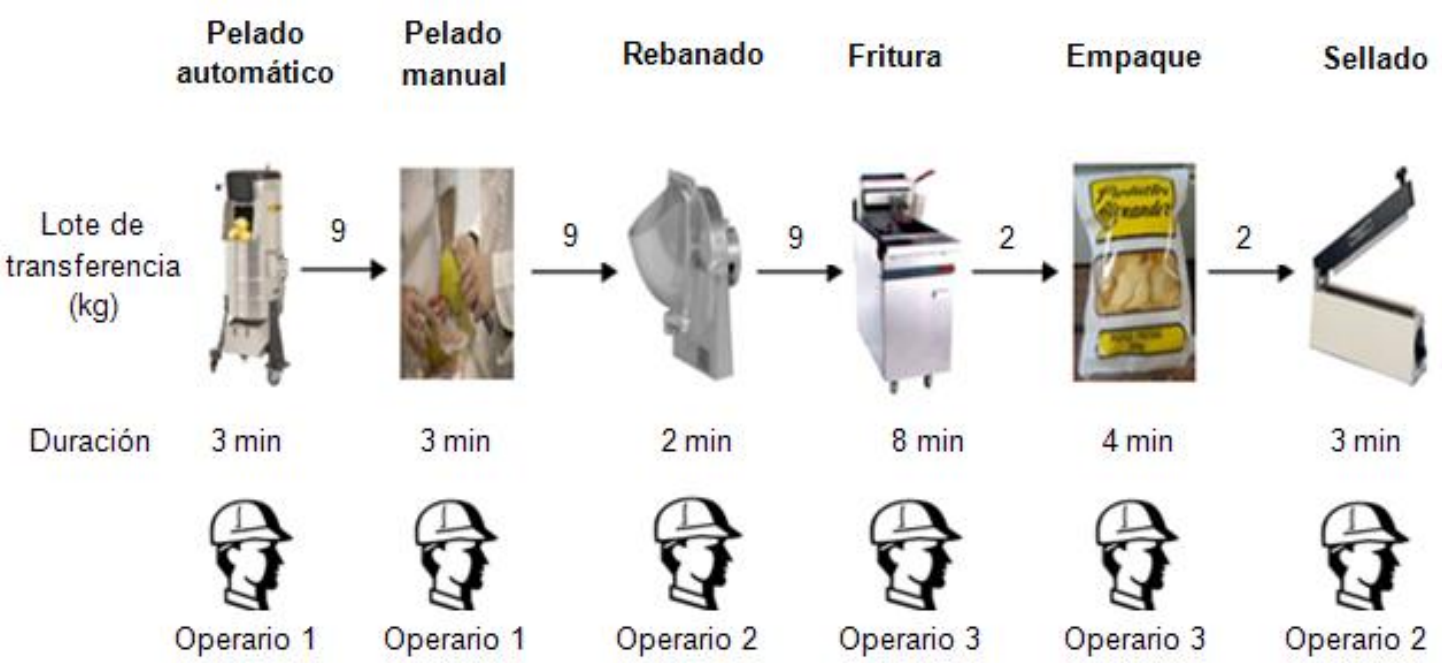


Figura 3. Propuesta de explotación de la restricción

Se ha logrado romper la restricción del sistema porque la producción aumenta en un $21 \%$ aproximadamente y permite cumplir el $100 \%$ de los pedidos en cantidad y tiempo con los clientes de la empresa.

Solo como complemento se continua con los siguientes pasos que plantea TOC, para la subordinación del sistema se propone que todos los procesos trabajen al ritmo de la restricción, es decir, a la capacidad de $1,20 \mathrm{~kg} /$ minuto que tiene la fritura en función de obtener un flujo estable para todos los procesos, evitar que inventario de producción en proceso se acumule frente a la restricción permitiendo así una mayor salida de producto.

Para elevar la restricción del sistema en caso de no lograr romperla en los pasos anteriores implicaría ya una inversión por parte de la empresa para la adquisición de una nueva freidora con la finalidad de que aliviane el trabajo acumulado en la restricción incrementando sin duda los niveles de producción.

Como se lograría un sistema totalmente flexible que podrá cumplir con todos los pedidos de los clientes y al mismo tiempo atender el aumento de demanda, se piensa que la restricción saltará ahora al mercado, y este inventario de producto terminado con el que contará el sistema debe ser introducido de manera inmediata al mercado a través de ofertas imposibles de rechazar evitando así pérdidas para la empresa y por el contrario perseguir la meta que ésta se ha planteado.

A continuación se muestra el Trúput que la empresa hubiera obtenido al cumplir con el 100\% de los pedidos, es decir, 4150 cajas de snacks de papas fritas en el año 2011.

$$
T=N(P V-C T V)
$$

Trúput actual: \$96811.98

Según datos de la empresa "Productos Alexander" el número de fundas dejadas de vender por restricciones del proceso fueron 1340 unidades.

Costo por unidad: $\$ 1.75$

$$
\begin{gathered}
T=1340(1.75-0.64) \\
T=1487.40 \\
T=96811.98+1487.40 \\
T=98299.38
\end{gathered}
$$

En base a lo anterior se puede afirmar que el Trúput en el año 2011 para la empresa trabajando en las restricciones resultó \$ 98299.38 . 
El Beneficio Neto en este período tomando en cuenta el nuevo Trúput calculado y los Gastos Operativos con un valor de $\$ 80907.27$ ya proporcionados por la empresa, excluyendo el costo de las tareas extra que ya no serían necesarias puesto que este aumento de la producción se lleva a cabo dentro de las 8 horas laborables y que más bien se considerarían como un ahorro de $\$$ 571.65 para la empresa en el año 2011.

$$
\begin{gathered}
\text { BENEFICIO NETO }=\text { TRÚPUT }- \text { GASTOSOPERATIVOS } \\
\text { BENEFICIO NETO }=98299.38-80907.24 \\
\text { BENEFICIO NETO }=17392.14
\end{gathered}
$$

En estas condiciones el Beneficio Neto obtenido es de \$17 392.14, es decir, \$2 059.05 más aplicando TOC.

\begin{tabular}{|l|c|c|}
\hline \multicolumn{1}{|c|}{ Indicadores de TOC } & Sin TOC & Aplicando TOC \\
\hline Trúput & 96811.98 & 98299.38 \\
\hline Gastos Operativos & 81478.89 & 80907.24 \\
\hline Beneficio Neto & 15333.09 & 17392.14 \\
\hline ROI & $33 \%$ & $36 \%$ \\
\hline
\end{tabular}

Con la aplicación de la Teoría de Restricciones el sistema sería más flexible para cumplir el 100\% de pedidos, podrá vender más productos y así aumentar su Beneficio Neto que es la meta de la empresa hoy y en el futuro.

\section{Conclusiones y recomendaciones}

- El diagnóstico del proceso de elaboración de snacks permitió determinar al proceso de fritura como el recurso con restricción de capacidad en el sistema debido al tiempo que se pierde en esta operación durante la jornada de trabajo y a la generación de pérdidas en tamaño y peso del producto.

- La explotación del recurso con restricción de capacidad buscó aprovechar al máximo el tiempo y la capacidad de producción, a través de una mejor programación de la producción, reducción de lote de transferencia de proceso a proceso, programación de la cantidad de trabajo para cada uno de los operarios en la jornada, preparación de procesos, fijación de turnos para el almuerzo de los operarios, todo esto en virtud de que la restricción siempre tenga trabajo y pueda llevarse a cabo de manera continua.

- A través de la explotación de la restricción, se programó una producción diaria de 51 lotes de $9 \mathrm{~kg}$ que se repiten en un intervalo de 8 minutos logrando aumentarla aproximadamente 
en un $21 \%$ para cumplir a cabalidad con los pedidos de la empresa, y lo más importante sin ningún tipo de inversión y sin generar egresos para la empresa.

- La propuesta presentada para la mejora continua del proceso productivo de elaboración de snacks utilizando como metodología la Teoría de Restricciones, una vez aplicada será capaz de aumentar la producción en planta y por lo tanto permitir el cumplimiento del $100 \%$ de los pedidos que recibe la empresa tanto en cantidad como en tiempos de entrega.

- El Beneficio Neto actual que genera el proceso de elaboración de snacks es de \$15 333.09 sin embargo la propuesta refleja que al aplicar la Teoría de Restricciones en el proceso de elaboración de snacks permitirá cumplir el $100 \%$ de los pedidos por lo que existirá un aumento de $\$ 2059.05$ en el beneficio lo que corresponde a lo que la empresa está perdiendo por incumplir dichos pedidos y se logrará obtener \$17392.14 de Beneficio Neto, y aunque no es una cantidad demasiado grande, hay que pensar que siempre hay algo que se puede mejorar.

\section{Bibliografía}

Agudelo L, Escobar J. (2010). Gestión por procesos. Medellín. Ed. Kimpres. P.237.

Aguerre, Miguel (2009). Teoría de restricciones. Recuperado el 25 de agosto de2011, de http://www.slideshare.net/cimaticdemexico/teoria-de-las-restricciones

Aguilar, Everardo y Vargas, Jaime (2008). Personas, economía y medio ambiente: las problemáticas de la pequeña empresa. Centro Regional de Investigación en Psicología,

Volumen 2, Número 1, Pág. 37-43.

Arias, J. (2006). Aplicación de los procesos de pensamiento (Teoría de Restricciones) al sistema educativo de la Universidad de Cuenca en la facultad de ciencias químicas.

Universidad de Cuenca facultad de ciencias químicas, 5. Recuperado el 27 de abril de 2012, de http://books.google.com.ec/books?id=RC7kpeEv1lAC\&pg=PA113\&dq=

Berrío, Deysi y Castrillón, Jaime (2008).Costos para gerenciar organizaciones manufactureras, comerciales y de servicio. (2da ed.). [En línea]. Editorial Uninorte. Recuperado el 24 de abril de 2012, de http://books.google.com.ec/books?id=HMgm0g4VopYC\&pg=PA186\&dq=\#v= onepage $\& q \& f=f a l s e$

Cruz, Carlos (2009). SMED. Recuperado el 10 de octubre de 2011, de http://industrial09.obolog.com/page/7

Espinoza, Carlos y Jiménez, Francisco. (2007). Costos industriales. (1a. ed.).[En línea]. Editorial Tecnológica de Costa Rica. Recuperado el 22 de agostode 2011, de 
http://books.google.com.ec/books?id=jRdhlWgPe60C\&pg=PA470\&dq=

Escalona, Iván. (2009). Teoría de Restricciones. Recuperado el 22 de agosto de 2011, de http://www.elprisma.com/apuntes/ingenieria_industrial/tocteoriadelasrestricciones/

Goldratt, E. y Cox, J. (1993). La meta. Un proceso de mejora continua. México: Ediciones North RiverPress. pp. 337.

Goldratt, E. (2002). Cómo Extraer Información del Océano de Datos. El Síndrome del Pajar. Quinta Ed. Ediciones Castillo. pp. 283.

Goldratt, E.(2009). La carrera: en busca de las ventajas competitivas. 1a ed. Buenos Aires: Granica. pp. 200

Herrera, I. (2003). Gestión moderna de producción aplicando la Teoría deRestricciones. Colombia: Artes Gráficas Tizan.

Iglesias, José. (2000). Un breve análisis de la teoría de las limitaciones. RevistaEspecial Encuentros A.E.C.A. Ibiza.

Krajewski L, Ritzman L, Malhotra M. (2008). Administración de operaciones. Octava edición. Pearson Ed. México. pp. 752.

Morales, J. (2006). Propuesta para implementar un sistema de programación de la producción, bajo Teoría de Restricciones, en una empresa de artes gráficas. Tesis no publicada, Universidad de Antioquia, Medellín, Colombia.

Viteri, J. Jacome, M. Beltrán, E. Competitividad e Innovación. Revista Economía y Negocios № 3. 50-55 pp. 